Z. Epileptol. 2021 · 34:251-252

https://doi.org/10.1007/s10309-021-00431-2

Angenommen: 21. Juni 2021

(c) Springer Medizin Verlag $\mathrm{GmbH}$, ein Teil von Springer Nature 2021

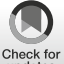

\title{
Telemedizin und mobile Gesundheitstechnologien
}

\section{Digitalisierung in der Epilepsieversorgung - Bedarf und Herausforderungen}

\author{
Sigrid Mues ${ }^{1} \cdot$ Rainer Surges ${ }^{2}$ \\ ${ }^{1}$ Ruhr Epileptologie, Klinik für Neurologie, Universitätsklinikum Knappschaftskrankenhaus Bochum, \\ Bochum, Deutschland \\ ${ }^{2}$ Klinik und Poliklinik für Epileptologie, Universitätsklinikum Bonn, Bonn, Deutschland
}

Liebe Leserin, lieber Leser!

„Digitalisierung soll im Alltag der Patienten ankommen", so Bundesgesundheitsminister Spahn im Juli 2020. Die Idee zu diesem Heft basiert jedoch nicht nur auf politische Ansagen und dem gespürten Trend zur Digitalisierung aller Lebensbereiche, sondern ist vielmehr der Tatsache geschuldet, dass wir bei der Versorgung von Menschen mit Epilepsie auch heute noch einer Reihe ungelöster fundamentaler Herausforderungen gegenüberstehen, die möglicherweise durch Einsatz neuer Gesundheitstechnologien und digitaler Anwendungen gelöst werden können.

Epilepsien sind durch spontan und wiederholt auftretende Anfälle charakterisiert. Dabei ist das Auftreten der Anfälle von den meisten Betroffenen nicht gut vorhersagbar [1]. Zudem geht aus Untersuchungen unter kontrollierten Bedingungen hervor, dass die Angaben zur Anfallshäufigkeit bei etwa zwei Drittel der Patientinnen und Patienten fehlerhaft sind, teilweise wurde mehr als die Hälfte aller Anfälle nicht wahrgenommen oder dokumentiert [2]. Bei Anfällen selbst kann es zu Störungen des Bewusstseins und zum Verlust der Körperkontrolle kommen, was natülich das Risiko für Unfälle und Verletzungen relevant erhöht. Generalisierte konvulsive Anfälle (also generalisierte oder fokal zu bilateral tonisch-klonische Anfälle) sind zudem mit SUDEP, dem plötzlichen unerwarteten Tod bei Epilepsie, assoziiert [3]. Von großer Bedeutung in der Versorgung von Menschen mit Anfallserkrankungen ist auch, dass trotz aller medizinischen Fortschritte der Anteil an falsch diagnostizierten Epilepsien in manchen Studien erschreckend hoch war und wir davon ausgehen müssen, dass ein beträchtlicher Anteil an Patientinnen und Patienten fälschlicherweise auf eine chronische Epilepsie behandelt werden [4]. Basierend auf diesen verschiedenartigen Herausforderungen lassen sich wichtige Anforderungen und Ziele ableiten, die in der medizinischen Praxis und Forschung mit Priorität angegangen werden sollten:

- Entwicklung von Technologien zur Warnung bei erhöhtem Risiko für Anfälle,

- Entwicklung von Technologien zur zuverlässigen automatischen Erkennung und Dokumentation aller Anfälle,

- Anwendung von Technologien zur Benachrichtigung von Hilfspersonen in Notfallsituationen,

- Anwendung und Verbreitung von Technologien zur Verbesserung der korrekten Stellung der Diagnose einer Epilepsie,

- Entwicklung und Anwendung von Apps zur Unterstützung in der Versorgung von Menschen mit Epilepsie,

- Sicherstellung einer breit zugänglichen, barrierefreien fachärztlichen telemedizinischen Beratung auf höchstem Niveau.

Der verbesserte Ausbau digitaler Infrastrukturen, die zunehmende Miniaturisierung hochleistungsfähiger Computer und Sensortechnologien und die breite Akzeptanz, solche Technologien tatsächlich im Alltag anzuwenden, könnten zur Lösung 
oben beschriebener Herausforderungen und Ziele beitragen und so Lebensqualität, Sicherheit und Lebenserwartung von Menschen mit Epilepsie merklich verbessern. Dazu werden aber auch hochwertige klinische Studien zur Prüfung mobiler Gesundheitstechnologien und Softwareanwendungen (Apps) benötigt, Hindernisse beim Aufbau und der Erweiterung telemedizinischer Beratungsmöglichkeiten müssen abgebaut und sachgerechte und angemessene Vergütungsmodalitäten für den Vertrieb mobiler Gesundheitstechnologien, Apps und telemedizinischer Beratungen vereinbart werden.

In dem vorliegenden Themenheft „Telemedizin und mobile Gesundheitstechnologien" greifen die Fachbeiträge verschiedene Aspekte oben genannter Herausforderungen und Ziele auf. So verschafft unser Beitrag „Umfrage zu Forschung und Entwicklung telemedizinischer Anwendungen und mobiler Gesundheitstechnologien bei Epilepsie in Deutschland, Österreich und der Schweiz" einen Überblick über das Spektrum aktueller Forschungsprojekte im deutschsprachigen Raum.

In der Übersichtsarbeit „Automatisierte Anfallsdetektion mit Wearables: Welche Technologien für welche Biosignale?" erläutern Andreas Schulze-Bonhage et al. den aktuellen Stand zu Grenzen und Möglichkeiten mobiler Gesundheitstechnologien, sog. Wearables, zur automatisierten Erkennung von Anfällen. Durch Wearables ist die kontinuierliche Erfassung epileptischer Anfälle möglich, was auch neue, vertiefende Kenntnisse zu Zyklen und Rhythmen bei Epilepsien liefern wird. Was man bereits über die Chronobiologie von Epilepsien weiß und wie man dieses Wissen zur Vorhersage eines erhöhten Anfallsrisikos und zu einer verbesserten Therapie nutzen könnte, erklärt Hermann Stefan in den beiden Artikeln zur "Chronodiagnostik in der Epileptologie" sowie "Chronopharmakologie und Chronotherapie bei Epilepsien".

Im weiteren Sinne zählen auch Smartphones zu mobilen Gesundheitstechnologien. Als täglicher Begleiter sind Smartphones prädestinierte Instrumente, die zur subjektiven Anfallsdokumentation, Verbesserung der Therapietreue und Kommunikationen mit ärztlichen Behandlerinnen und Behandlern beitragen können. Dazu berichten Sarah von Spiczak et al. in ihrem Beitrag „Mobile Apps und Internet-Anwendungen zur Unterstützung von Versorgung und Dokumentation bei Epilepsie". Auch schon längst zum ärztlichen Alltag gehört das Ansehen von kurzen Filmsequenzen von Anfällen, die von Angehörigen oder Freunden mit dem Smartphone aufgezeichnet wurden. Wie Smartphonevideos zur korrekten Diagnosestellung beitragen und wie das Aufzeichnen solcher Filme verbessert werden könnte, erklären Stephanie Rammé et al. in dem Artikel „Handyvideos als diagnostisches Instrument anfallsartig auftretender Ereignisse". Auch die Analyse von Videos kann in der Versorgung und Sicherheit von Menschen mit Epilepsie z. B. in Einrichtungen und Kliniken relevant verbessern. Den aktuellen Stand hierzu fassen Peter Hopp und Thomas Mayer in „Bedeutung Video-basierter Anfallsdetektion" zusammen.

Auf verschiedene Aspekte zur Telemedizin wird in fünf Fachbeiträgen näher eingegangen. Den aktuellen Stand zu Anwendung und Perspektiven einer kollegialen ärztlichen Beratung erläutern Sigrid Mues et al. in der Übersichtsarbeit „Telemedizin in der Epilepsieversorgung: Arzt-zu-Arzt-Anwendungen. Teil I: State-of-the-Art, Herausforderungen, Perspektiven". In Teil II: Aktuelle Projekte in Deutschland werden fünf aktuelle Projekte dargestellt. Schließlich erläutern Randi von Wrede mit Rainer Surges die Besonderheiten telemedizinischer „Patient-zu-Arzt-Anwendungen in der ambulanten Epilepsieversorgung". Die Originalarbeiten „Tele-Epileptologie Ruhr (TE Ruhr): Zwischenevaluation eines telemedizinischen Modellprojektes" von Sigrid Mues et al. und „Einstellungen und Hinderungsgründe bezüglich Telemedizin bei Epilepsien: Eine Umfrage in neurologischen Praxen" von Johann Philipp Zöllner et al. untersuchen die Herausforderungen bei der praktischen Umsetzung telemedizinischer Beratungsangebote.

Wir finden, dass dieses Themenheft die Vielfältigkeit und das Potenzial der Digitalisierung in der Versorgung von Menschen mit Epilepsie widerspiegelt und zeigt, dass zumindest manche Aspekte der Digitalisierung tatsächlich auch schon im Alltag angekommen sind. Wir danken allen Autorinnen und Autoren für die gelungenen Beiträgen, dem Verlag für die unkomplizierte Zusammenarbeit und den beiden Herausgebern für die Möglichkeit, diese besondere Ausgabe zu realisieren.

Ihnen nun, liebe Leserin, lieber Leser, wünschen wir eine unterhaltsame und informative Lektüre beim Themenheft "Telemedizin und mobile Gesundheitstechnologien".

Sigrid Mues

Rainer Surges

\section{Korrespondenzadresse}

\section{Dr. med. Sigrid Mues}

Ruhr Epileptologie, Klinik für Neurologie, Universitätsklinikum Knappschaftskrankenhaus Bochum

In der Schornau 23-25, 44892 Bochum,

Deutschland

sigrid.mues@kk-bochum.de

Univ.-Prof. Dr. med. Rainer Surges, MHBA Klinik und Poliklinik für Epileptologie, Universitätsklinikum Bonn

Venusberg-Campus 1, 53127 Bonn, Deutschland

rainer.surges@ukbonn.de

Interessenkonflikt. R. Surges hat in den letzten 3 Jahren Honorare für Vorträge oder als Berater von Arvelle, Angelini, Bial, Desitin, Eisai, LivaNova, Novartis, UCB Pharma und UnEEG erhalten. S. Mues gibt an, dass kein Interessenskonflikt besteht.

\section{Literatur}

1. Privitera M, Haut SR, Lipton RB, McGinley JS, Cornes $S$ (2019) Seizure self-prediction in a randomized controlled trial of stress management. Neurology 93:e2021-e2031

2. Hoppe C, Poepel A, Elger CE (2007) Epilepsy: accuracy of patient seizure counts. Arch Neurol 64:1595-1599

3. Surges $R$, Conrad $S$, Hamer HM, Schulze-Bonhage A, Staack AM, Steinhoff BJ, Strzelczyk A, Trinka E (2021) SUDEP kompakt - praxisrelevante Erkenntnisse und Empfehlungen zum plötzlichen, unerwarteten Tod bei Epilepsie. Nervenarzt. https://doi.org/10.1007/s00115-021-01075-3

4. Xu Y, Nguyen D, Mohamed A, Carcel C, Li Q, Kutlubaev MA, Anderson CS, Hackett ML (2016) Frequency of a false positive diagnosis of epilepsy: a systematic review of observational studies. Seizure 41:167-174 